\title{
Efficacy of Bamlanivimab/Etesevimab and Casirivimab/Imdevimab in Preventing Progression to Severe COVID-19 and Role of Variants of Concern
}

\author{
Marco Falcone - Giusy Tiseo • Beatrice Valoriani · Chiara Barbieri • \\ Sara Occhineri - Paola Mazzetti - Maria Linda Vatteroni - Lorenzo Roberto Suardi • \\ Niccolò Riccardi · Mauro Pistello · Danilo Tacconi · Francesco Menichetti
}

Received: July 6, 2021 / Accepted: August 10, 2021 / Published online: August 25, 2021

(C) The Author(s) 2021

\section{ABSTRACT}

Introduction: The aim of this study was to evaluate the risk of hospitalization or death in patients infected by SARS-CoV2 variants of concern (VOCs) receiving combinations of monoclonal antibodies (mAbs), bamlanivimab/ etesevimab or casirivimab/imdevimab.

Methods: Observational prospective study conducted in two Italian hospitals (University Hospital of Pisa and San Donato Hospital, Arezzo) including consecutive outpatients with COVID-19 who received bamlanivimab/etesevimab or casirivimab/imdevimab from March

Supplementary Information The online version contains supplementary material available at https:// doi.org/10.1007/s40121-021-00525-4.

M. Falcone $(\bowtie) \cdot$ G. Tiseo $\cdot$ C. Barbieri $\cdot$ S. Occhineri . L. R. Suardi - N. Riccardi - F. Menichetti Infectious Diseases Unit, Department of Clinical and Experimental Medicine, Azienza Ospedaliera Universitaria Pisana, University of Pisa, Via Paradisa 2, 56124 Pisa, Italy

e-mail: marco.falcone@unipi.it

B. Valoriani · D. Tacconi

Infectious Disease Unit, San Donato Hospital Arezzo, Arezzo, Italy

P. Mazzetti · M. L. Vatteroni · M. Pistello Virology Unit, Pisa University Hospital, Pisa, Italy

M. Pistello

Retrovirus Center, Department of Translational

Research, University of Pisa, Pisa, Italy 20th to May 10th 2021. All patients were at high risk of COVID-19 progression according to FDA/AIFA recommendations. Patients were divided into two study groups according to the infecting viral strain (VOCs): Alpha and Gamma group. The primary endpoint was a composite of hospitalization or death within 30 days from mAbs infusion. A Cox regression multivariate analysis was performed to identify factors associated with the primary outcome in the overall population.

Results: The study included 165 patients: 105 were infected by the VOC Alpha and 43 by the VOC Gamma. In the Alpha group, no differences in the primary endpoint were observed between patients treated with bamlanivimab/ etesevimab or casirivimab/imdevimab. Conversely, in the Gamma group, a higher proportion of patients treated with bamlanivimab/ etesevimab met the primary endpoint compared to those receiving casirivimab/imdevimab (55\% vs. $17.4 \%, p=0.013)$. On multivariate Cox-regression analysis, the Gamma variant and days from symptoms onset to mAbs infusion were factors independently associated with higher risk of hospitalization or death, while casirivimab/imdevimab was protective (HR 0.33, 95\% CI 0.13-0.83, $p=0.019$ ).

Conclusions: In patients infected by the SARSCoV-2 Gamma variant, bamlanivimab/etesevimab should be used with caution because of the high risk of disease progression. 
Keywords: Bamlanivimab/etesevimab; Casirivimab/imdevimab; COVID-19; Monoclonal antibodies; SARS-CoV-2; Variants of concern

\section{Key Summary Points}

\section{Why carry out this study?}

The spread of SARS-CoV-2 variants are raising concerns because of their extensive mutations in the spike protein that could lead to antigenic changes detrimental to mAbs therapies and vaccine protection.

Little is known about the efficacy of the two mAbs casirivimab/imdevimab and bamlanivimab/etesevimab against SARS$\mathrm{CoV}-2$ variants of concern.

\section{What was learned from the study?}

In patients with the Gamma variant, casirivimab/imdevimab should be preferred because it is associated with a reduced risk of disease progression.

The knowledge of SARS-CoV-2 variant may allow a more appropriate use of mAbs.

Further clinical studies are warranted to evaluate the efficacy of the bamlanivimba/etesevimab and casirivimab/imdevimab against the Delta variant.

\section{INTRODUCTION}

COVID-19 continues to represent a significant cause of hospitalization and death [1-3], despite the availability of vaccines and the use of different treatments $[4,5]$. Neutralizing monoclonal antibodies (mAbs) developed from convalescent COVID-19 patients target the surface spike (S) glycoprotein that mediates viral entry into host cells [6], and represent a promising therapeutic option in the early phase of COVID-19. Several human mAbs targeting the SARS-CoV-2 are currently being studied in clinical trials, and the US Food and Drug Administration issued an emergency use authorization of some mAbs for the treatment of outpatients with mild-to-moderate COVID19. Bamlanivimab (LY-CoV555; Eli Lilly)/etesevimab (LY-CoV016; Eli Lilly), and casirivimab/ imdevimab (REGN-COV2; Regeneron Pharmaceuticals) have been demonstrated to reduce the viral load in Phase 3 trials $[7,8]$; and recent releases reported a reduced risk of hospitalization or death in high-risk non-hospitalized patients with COVID-19 [9].

Variants of concern (VOCs) identified in the United Kingdom (Alpha), South Africa (Beta), and Brazil (Gamma) have been associated with higher transmissibility and disease severity, and have potential implications for diagnostics and effectiveness of currently available mAbs [10]. Preclinical studies showed that viral entry driven by the $S$ protein of the Beta and Gamma variants is inhibited by imdevimab, but resistant to casirivimab and bamlanivimab [11].

The aim of this study is to evaluate the clinical efficacy of bamlanivimab/etesevimab and casirivimab/imdevimab against Alpha (also known as B.1.1.7, 20I/501Y.V1, VOC 202012/01) and Gamma (P.1, 20J/501Y.V3, VOC-202101/02) variants in outpatients with COVID-19 at high risk of disease progression.

\section{METHODS}

\section{Study Population}

This is an observational study conducted in two hospitals (Azienda Ospedaliera Universitaria Pisana and San Donato Hospital, Arezzo) in Italy from March 20th 2021 to June 10th 2021. Patients were eligible for inclusion in this study if they were consecutive outpatients with documented COVID-19 by antigen or reverse transcriptase-polymerase chain reaction (RT-PCR) test on a nasopharyngeal swab, and treated with mAbs targeting SARS-CoV-2. We included patients treated with mAbs from March 20th 2021 to May 10th 2021: Follow-up continued until 10th June 2021 (completion of 30 days 
follow-up of the last treated patient). This analysis focuses on comparative outcomes of patients infected with VOCs who received bamlanivimab/etesevimab and those who received casirivimab/imdevimab.

The study was conducted according to the principles stated in the Declaration of Helsinki, and approved by the local Ethical Committee of the Area Vasta Nord Ovest of Tuscany region (IRB number 230320). All patients signed a written informed consent.

\section{Monoclonal Antibodies Exposure}

Patients received bamlanivimab (700 mg) combined with etesevimab (1400 mg) or casirivimab $(1200 \mathrm{mg})$ combined with imdevimab (1200 mg). Indications for receiving mAbs, according to AIFA recommendations [12], were: (1) mild-to-moderate COVID-19, (2) recent infection (symptoms for no more than 10 days), and (3) high risk of progression to severe disease or hospitalization (body mass index $\geq 35 \mathrm{Kg} / \mathrm{m}^{2}$, hemodialysis, diabetes with $\mathrm{HbA1c}>9 \%$, immunosuppressive disease/immunosuppressive treatment, $\geq 55$ years old, and concomitant cardiovascular disease, or hypertension, or chronic obstructive pulmonary disease/other chronic respiratory disease). Exclusion criteria were hospitalization for COVID-19, need for oxygen-therapy due to COVID-19, or requirement of an increase in baseline oxygen flow rate due to COVID-19 in those on chronic oxygen therapy due to underlying non-COVID-19-related comorbidities.

\section{Outcomes}

The aim of this study was to evaluate the clinical efficacy of bamlanivimab/etesevimab and casirivimab/imdevimab in patients infected with the Alpha or Gamma variants. The primary endpoint was a composite of hospitalization or death within 30 days from mAbs infusion. The main exposure variable was the anti-SARS-CoV2 mAbs combination: bamlanivimab/etesevimab or casirivimab/imdevimab.

Secondary outcomes included intensive care unit (ICU) admission and time to virological cure (days from mAbs infusion and negative nasopharyngeal swab).

\section{Study Procedures}

Epidemiological and demographic information, medical history, comorbidities, and information on clinical symptoms were prospectively collected. Patients underwent weekly ambulatory visits during which physical examination and nasopharyngeal swab were performed. Clinical cure was defined as resolution of symptoms and RT-PCR negative nasopharyngeal swab test.

Characterization of the VOC Alpha (B.1.1.7, UK), Gamma (P.1, Brazil), and Beta (501Y.V2, South Africa) was performed in two steps. SARSCoV-2 RNA-positive swabs were preliminarily screened with TaqPath COVID-19 CE-IVD RTPCR Kit (ThermoFisher Scientific, Monza, Italy) that detects the $S$ gene mutations HV 69-70del typical of the Alpha variant, or COVID-19 Variant Catcher (Clonit, Pavia, Italy) that, in addition to HV 69-70del, identifies $\mathrm{E} 484 \mathrm{~K}$ and $\mathrm{N} 501 \mathrm{Y}$ mutations. Isolates not belonging to the Gamma variant were sequenced in the $S$ region, nt 1311-2225, to search for mutations E484K, N501Y, D614G, and H655Y to identify the P.1 variant. Sequences were deposited in GISAID and are available on request.

\section{Statistical Analysis}

Continuous variables are reported as mean $\pm s$ tandard deviation and medians and interquartile ranges (IQRs) according to their distribution. The normality of distributions was assessed by the Kolmogorov-Smirnov test. Continuous variables were compared by the Student's $t$ test or the Mann-Whitney $U$ test, as appropriate. Categorical data were expressed as frequency distributions, and the $\chi^{2}$ test or Fisher's exact test was used to determine whether differences existed between groups.

The study population was divided into two groups: patients infected with the Alpha variant and those infected with the Gamma variant. In each group, the primary endpoint in patients treated with bamlanivimab/etesevimab versus 
those treated with casirivimab/imdevimab was calculated. Kaplan-Meier curves were built in the two study groups to evaluate the time-dependent risk of the primary endpoint according to the type of mAbs combination.

A multivariate Cox regression analysis was performed to identify factors associated with the primary endpoint. Multivariate analysis using logistic regression prediction models was constructed using a forward stepwise procedure, entering all variables with univariate $p<0.05$ and those deemed clinically significant. The final multivariate model was chosen according to the Akaike information criterion and to parsimony and clinical interpretability of the data. Statistical significance was established at $p<0.05$. All reported $p$ values are two-tailed. The results obtained were analyzed using a commercially available statistical software package (SPSS 20.0; IBM, Armonk, NY, USA, and R 3.5.1, Vienna, Austria).

\section{RESULTS}

A total of 221 patients were treated in the two study centers. Ninety-one (41.2\%) received bamlanivimab/etesevimab and 123 (55.6\%) casirivimab/imdevimab (Fig. 1). Seven (3.2\%) received bamlanivimab monotherapy and were excluded from comparative analysis. Eighteen patients in the bamlanivimab/etesevimab group and 31 in the casirivimab/imdevimab group were infected by the wild-type variant. Characteristics of patients infected with the wild-type variant are reported in Supplementary Table 1. In this group of patients, no differences in hospitalization or death were observed between patients treated with bamlanivimab/etesevimab and those who received casirivimab/imdevimab $(n=1 / 18,5.6 \%$ vs. $n=4 / 31,12.9 \%, p=0.413)$.

The remaining 165 patients were infected by VOCs: of these, 73 were treated with bamlanivimab/etesevimab (53 with Alpha and 20 with Gamma variants) and 92 with casirivimab/ imdevimab (69 infected with Alpha and 23 with Gamma variants). All patients completed 30-day follow-up. There were no patients lost to follow-up.

As shown in Table 1, there were no differences in age, comorbidities, and previous antiSARS CoV-2 vaccination between patients who received bamlanivimab/etesevimab and those who received casirivimab/imdevimab. Patients who received casirivimab/imdevimab were more frequently obese subjects.

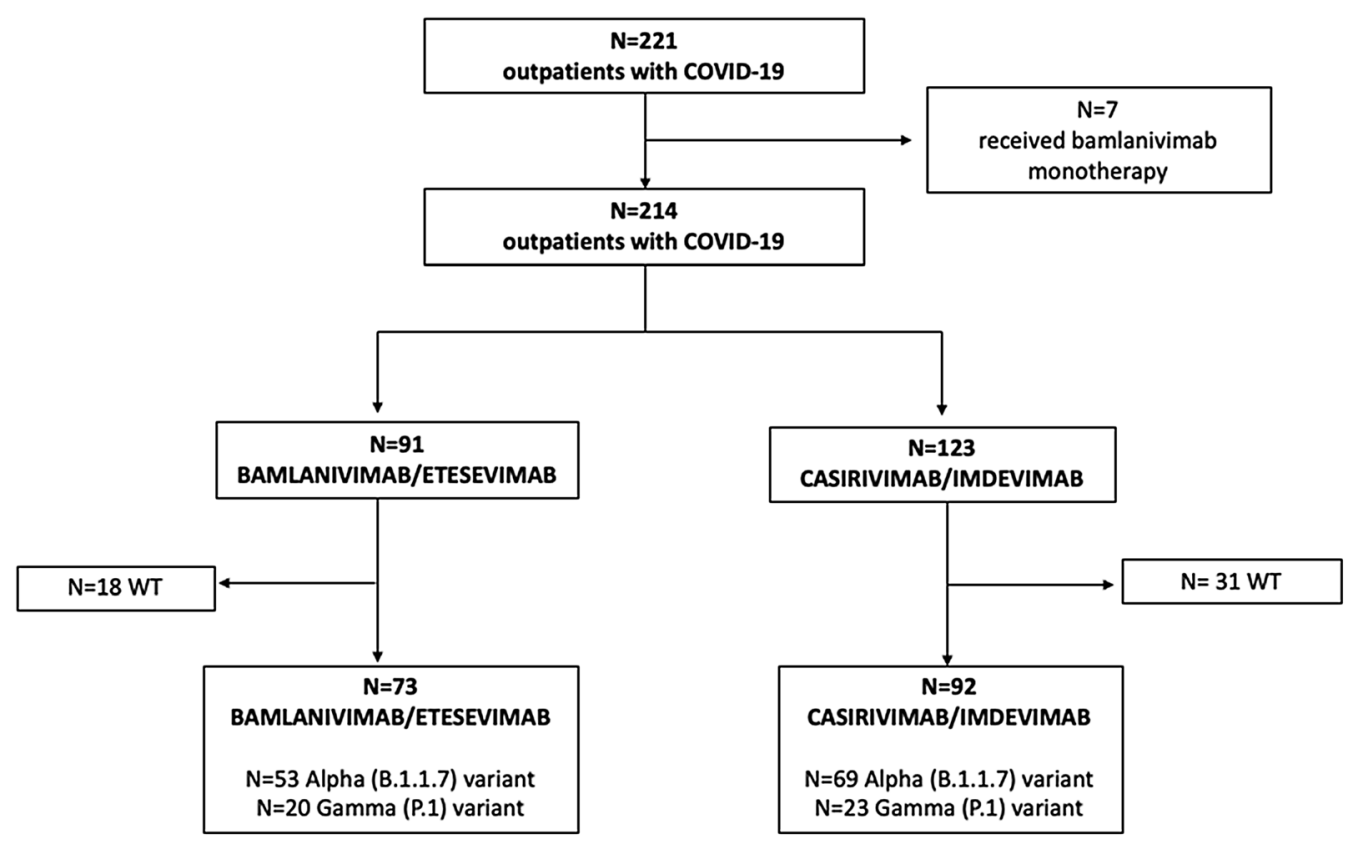

Fig. 1 Study flow chart. WT wild-type 
Table 1 Comparison of patients treated with bamlanivimab/etesevimab and those treated with casirivimab/imdevimab

\begin{tabular}{|c|c|c|c|}
\hline & $\begin{array}{l}\text { Balmanivimab/ } \\
\text { etesevimab, } n=73(\%)\end{array}$ & $\begin{array}{l}\text { Casirivimab/ } \\
\text { imdevimab, } n=92(\%)\end{array}$ & $p$ value \\
\hline Age, median (IQRs), years & $69(58-77.5)$ & $65(56.25-75.75)$ & 0.166 \\
\hline Male sex & $37(50.7)$ & $44(47.8)$ & 0.715 \\
\hline \multicolumn{4}{|l|}{ Comorbidities } \\
\hline COPD & $9(12.3)$ & $16(17.4)$ & 0.368 \\
\hline Diabetes mellitus & $8(11)$ & $14(15.2)$ & 0.570 \\
\hline Cardiovascular disease & $43(58.9)$ & $51(55.4)$ & 0.655 \\
\hline Chronic renal failure & $3(4.1)$ & $3(3.3)$ & 1.0 \\
\hline Solid cancer & $4(5.5)$ & $7(7.6)$ & 0.756 \\
\hline Hematological malignancy & $5(6.8)$ & $4(4.3)$ & 0.511 \\
\hline Solid organ/bone marrow transplantation & $3(4.1)$ & $1(1.1)$ & 0.323 \\
\hline Obesity & $4(5.5)$ & $15(16.3)$ & 0.047 \\
\hline Autoimmune disease & $7(9.6)$ & $10(10.9)$ & 1.0 \\
\hline Charlson comorbidity index, median (IQRs) & $2(1-4)$ & $2(1-3)$ & 0.226 \\
\hline $\begin{array}{l}\text { Days from onset of symptoms to monoclonal } \\
\text { antibodies infusion, median (IQRs) }\end{array}$ & $4(3-5.75)$ & $5(3-7)$ & 0.105 \\
\hline Previous anti-SARS-CoV-2 vaccination & $16(21.9)$ & $28(30.4)$ & 0.219 \\
\hline mRNA-1273 (Moderna) & $5(6.8)$ & $9(9.8)$ & \\
\hline BNT162b2 (Pfizer) & $7(9.6)$ & $16(17.4)$ & \\
\hline ChAdOx1 nCoV-19 (Astrazeneca) & $4(5.5)$ & $3(3.3)$ & \\
\hline Alpha (B.1.1.7) variant & $53(72.6)$ & $69(75)$ & 0.728 \\
\hline Gamma (P.1) variant & $20(27.4)$ & $23(25)$ & 0.728 \\
\hline Hospitalization & $13(17.8)$ & $6(6.5)$ & 0.029 \\
\hline ICU admission & $3(4.1)$ & 0 & 0.085 \\
\hline 30-day mortality & $3(5)$ & $1(1.8)$ & 0.619 \\
\hline Time to virological cure, days, median (IQRs) ${ }^{a}$ & $15(10-21)$ & $15.5(11-27.5)$ & 0.361 \\
\hline
\end{tabular}

Bold italics indicates the statistical significance $(p<0.05)$

$C O P D$ chronic obstructive pulmonary disease, ICU intensive care unit, IQRs interquartile ranges

a Time from monoclonal antibodies infusion and virological cure was calculated after excluding patients who died

Table 2 shows primary and secondary endpoints according to VOC and type of mAbs combination. In the group of patients infected with the Alpha variant, the $5.7 \%(3 / 53)$ of patients who received bamlanivimab/etesevimab and the $4.3 \%(3 / 69)$ of patients who received casirivimab/imdevimab met the primary endpoint $(p=1.0)$. Conversely, in the group of patients infected with the Gamma variant, a higher proportion of patients treated with bamlanivimab/etesevimab met the primary endpoint compared to those who received 
Table 2 Primary and secondary endpoints according to exposure variable (bamlanivimab/etesevimab or casirivimab/ imdevimab) in patients with Alpha and Gamma VOCs

\begin{tabular}{lclc}
\hline & \multicolumn{1}{c}{ Balmanivimab/etesevimab } & Casirivimab/imdevimab & $\boldsymbol{p}$ value \\
\hline $\begin{array}{l}\text { Primary endpoint } \\
\text { Composite of hospitalization or death, } n(\%)\end{array}$ & & \\
Alpha (B.1.1.7) variant & $3 / 53(5.7 \%)$ & $3 / 69(4.3 \%)$ & 1.0 \\
Gamma (P.1) variant & $11 / 20(55 \%)$ & $4 / 23(17.4 \%)$ & $\mathbf{0 . 0 1 3}$ \\
Secondary endpoints & & & \\
ICU admission, $n$ (\%) & $0 / 53$ & $0 / 69$ & - \\
Alpha (B.1.1.7) variant & $3 / 20(15 \%)$ & $0 / 23$ & 0.092 \\
Gamma (P.1) variant & & 0.834 \\
Time to virological cure, median (IQRs) & $15(9-21)$ & $\boldsymbol{0 . 0 4}$ \\
Alpha (B.1.1.7) variant & $14(10.5-22.5)$ & $14(0-16)$ & \\
Gamma (P.1) variant & $17(12.5-30)$ & & \\
\hline
\end{tabular}

Bold italics indicates the statistical significance $(p<0.05)$

$I C U$ intensive care unit

casirivimab/imdevimab $\quad(n=11 / 20, \quad 55 \%$ vs. $n=4 / 23,17.4 \%, p=0.013)$. Among patients infected with the Gamma varient, time from $m A b s$ infusion to virological cure was longer in patients treated with bamlanivimab/etesevimab (median 17 days, IQRs 12.5-30) compared to those who received casirivimab/imdevimab (median 14 days, IQRs $0-16, p=0.04$ ).

Figure 2 shows the probability of hospitalization-free survival of patients who received bamlanivimab/etesevimab versus those who received casirivimab/imdevimab in the subgroup of patients infected with the Alpha VOC (Panel A) and with the Gamma VOC (Panel B). In patients with Gamma VOC, bamlanivimab/ etesevimab was associated with a lower risk of hospitalization-free survival $(p=0.016, \log$ rank).

Comparison of patients who met the primary endpoint and those who did not is reported in Supplementary Table 2. On multivariate Cox regression analysis, the Gamma variant (HR 9.84, 95\% CI 3.74-25.88, $p<0.001$ ) and days from onset of symptoms to mAbs infusion (HR 1.36, 95\% CI 1.1-1.66, $p=0.003$ ) were factors independently associated with higher risk of hospitalization or death. In contrast, casirivimab/imdevimab was a protective factor (HR 0.33, 95\% CI 0.13-0.83, $p=0.019$ ) (Table 3).

\section{DISCUSSION}

The spread of SARS-CoV-2 variants Alpha, Gamma, and Delta are raising concerns not only for their increased transmissibility but also because of their extensive mutations in the spike protein that could lead to antigenic changes detrimental to mAbs therapies and vaccine protection. This is the first clinical study reporting the clinical efficacy of the two authorized anti-SARS CoV-2 mAbs combinations, bamlanivimab/etesevimab and casirivimab/imdevimab, in patients infected with different VOCs.

The main finding of our study is that patients infected by the Gamma variant treated with bamlanivimab/etesevimab had a higher risk of hospitalization or death compared to those who received casirivimab/imdevimab. This observation is in line with evidence from in vitro studies [11, 13, 14]. Hoffman et al. investigated whether casirivimab/imdevimab 


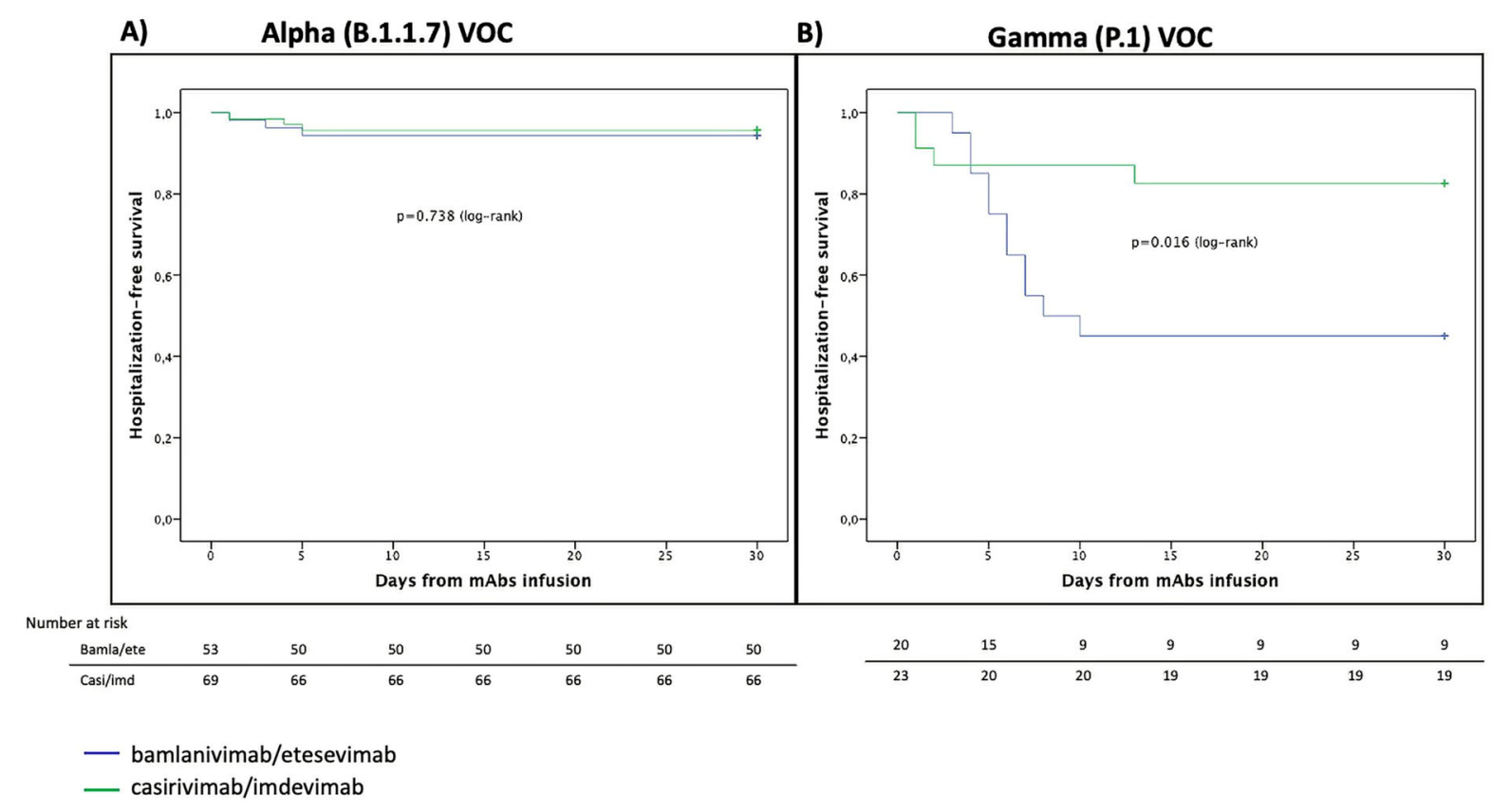

Fig. 2 Survival curves of patients with the Alpha (A) and those with the Gamma (B) VOC according to exposure antibodies, Bamla/ete bamlanivimab/etesevimab, Casi/ variable (bamlanivimab/etesevimab or casirivimab/imdeimd casirivimab/imdevimab vimab). $V O C$ variant of concern, $m A b s$ monoclonal

Table 3 Multivariate Cox regression analysis of factors independently associated with primary endpoint (hospitalization or death within 30 days from monoclonal antibodies infusion)

\begin{tabular}{llll}
\hline & HR (95\% CI) & $\boldsymbol{p}$ value \\
\hline Gamma (P.1) variant & $9.84(3.74-25.88)$ & $<\boldsymbol{0 . 0 0 1}$ \\
Time from symptoms onset to monoclonal antibodies infusion (each-day increment) & $1.36(1.1-1.66)$ & $\mathbf{0 . 0 0 3}$ \\
Casirivimab/imdevimab & $0.33(0.13-0.83)$ & $\boldsymbol{0 . 0 1 9}$ \\
\hline
\end{tabular}

Bold italics indicates the statistical significance $(p<0.05)$

and bamlanivimab inhibit the viral entry of the Alpha, Beta, and Gamma variants; they found that the casirivimab/imdevimab combination was efficient in inhibiting entry mediated by the $S$ proteins of all variants, while bamlanivimab failed to inhibit viral entry of the Beta and Gamma variants [11]. Furthermore, the $\mathrm{E} 484 \mathrm{~K}$ mutation involving the spike protein (typical of the Gamma variant) results in a marked reduction in in vitro susceptibility to bamlanivimab [9], and the Gamma variant itself was found not only to be less susceptible to multiple neutralizing monoclonal antibodies but also to neutralization by convalescent plasma and vaccine sera [13, 14]. The B.1.617.2 (Delta) variant of SARS-CoV-2 is currently widespread. It was identified in October 2020 in India and then became dominant in the UK and further spread to many countries. Evidence suggests that it is potentially more transmissible than other variants. Recent data have shown that the Delta variant is resistant to neutralization by some anti-NTD and anti-RBD mAbs including bamlanivimab [15]. Our study was conducted before the spread of the Delta variant in Europe. Thus, we were not able to evaluate 
the role of the two mAbs combinations against this later VOC. Further clinical studies are warranted to evaluate the efficacy of different mAbs against the Delta variant.

Our findings suggest the importance of viral sequencing for therapeutic purposes. In recent months, four viral lineages reflecting VOCs have emerged and merit close monitoring: Alpha, Beta, Gamma, and Delta. The Alpha variant, first reported in the United Kingdom [16], is the dominant variant in several countries all over the world [17]. Unfortunately, sequencing capacity varies greatly across different countries. In Europe, only 12 countries met the recommended level of $10 \%$ SARS-CoV-2positive cases sequenced [18]. In a recent surveillance study, among 3.2 million COVID19 cases reported from several European countries, only 23,343 had information on SARSCoV-2 variants, and the vast majority (85.6\%) were VOCs [19].

The European Centre for Disease Prevention and Control showed that, between 28 June and 11 July 2021, the Delta variant was dominant in the majority (19 countries) of the 28 European countries that reported sufficiently complete genetic sequencing information [20]. In Italy, the reported prevalence of the Delta variant of SARS-CoV-2 on 20 July was $94.8 \%$, with values ranging between 80 and $100 \%$ in the individual regions. On the same date, the Alpha variant had a prevalence of $3.2 \%$ (with a range between 0 and $14.7 \%$ ), while the Gamma variant was $1.4 \%(0-16,7 \%)[21]$. In the Tuscany region, the Delta variant now represents over $90 \%$ of the strains sequenced [21].

In our study population, including patients treated from March to May 2021, the Gamma variant was significantly represented. Thus, a rapid screening for the Gamma variant may be useful in some epidemiological contexts because it may guide the choice of mAbs combination. We also found that casirivimab/ imdevimab is independently associated with better clinical outcome, even after controlling for other factors, such as the Gamma variant and the time from onset of symptoms. To our knowledge, no data on the efficacy of mAbs from real life are available. We observed that casirivimab/imdevimab is independently associated with a 73\% of risk reduction of hospitalization or death. This finding is in line with the last evidence from the RECOVERY randomized clinical trial [8]. In this trial, hospitalized patients with COVID-19 were randomly allocated $(1: 1)$ to either the usual standard of care alone or the usual care plus a single dose of casirivimab $4 \mathrm{~g}$ and imdevimab $4 \mathrm{~g}$ by intravenous infusion; this mAbs combination significantly reduced 28-day mortality among patients who were seronegative at baseline [9]. The ongoing evidence from the literature seems to confirm the beneficial effect of mAbs in improving the outcome of patients with COVID-19.

This study has several limitations. First, the sample size is not large, and so our findings need to be confirmed on a large scale; however, to date, very limited data about the clinical use of mAbs are available, and our series represents one of the largest published in the literature. Furthermore, our findings may be relevant in a context of high incidence of VOCs, but may be less generalizable to other epidemiological settings. The knowledge of SARS-CoV-2 variants before mAbs infusion is not feasible. Considering the weak molecular epidemiology surveillance in Italy, the allocation to bamlanivimab/ etesevimab or casirivimab/imdevimab is difficult to perform based on surveillance data. Since recent data have shown that a reduction of the spread of the Alpha variant in favor of the Delta variant in most Italian regions, the use of casirivimab/imdevimab instead of bamlanivimab/imdevimab seems to be more prudent. Further clinical data to evaluate the efficacy of casirivimab/imdevimab on the Delta variant are needed. Finally, since this was an observational (non-randomized) study, the choice to administer bamlanivimab/etesevimab or casirivimab/imdevimab was made according to the prescriber's judgment, leading to a selection bias. However, we can acknowledge that the choice was made according to the drug availability and not to patients' clinical conditions. In fact, the two groups of patients were well balanced and no differences in the severity of illness were detected. 


\section{CONCLUSION}

The two authorized anti-SARS-CoV-2 mAbs combinations, bamlanivimab/etesevimab and casirivimab/imdevimab, may have different efficacy against VOCs. Compared to casirivimab/imdevimab, bamlanivimab/etesevimab seems to be associated with an increased risk of hospitalization or death in patients infected with the Gamma variant. The knowledge of infecting SARS-CoV-2 variant may allow a more appropriate use of mAbs.

\section{ACKNOWLEDGEMENTS}

We thank the participants of the study.

Funding. No funding or sponsorship was received for this study or publication of this article. The Rapid Service Fee was funded by the authors.

Authorship. All named authors meet the International Committee of Medical Journal Editors (ICMJE) criteria for authorship for this article, take responsibility for the integrity of the work as a whole, and have given their approval for this version to be published.

Authorship contributions. MF, DT and FM concepted and designed the study; GT, BV, CB, LRS, NR and SO collected data; GT performed the statistical analysis; MF and GT drafted the manuscript; PM, MLV, and MP performed the sequencing of the variants. MF, MP and FM revised the manuscript. All authors contributed to the critical revision of the final manuscript.

Disclosures. Falcone $\mathrm{M}$ received grants and speaker honoraria from Angelini, Shionogi, Gilead and Nordic Pharma. Menichetti F has participated in advisory boards and/or received speaker honoraria from Angelini, Correvio, Merck Sharp \& Dohme (MSD), Nordic Pharma, Pfizer, Astellas, Gilead, Bristol-Myers Squibb (BMS), Janssen, ViiV, bioM rieux, Biotest, Becton Dickinson, Pfizer, and Shionogi. Tiseo G, Valoriani B, Barbieri C, Occhineri S, Mazzetti P,
Vatteroni ML, Pistello M, Tacconi D have nothing to disclose. All reported disclosures are outside this study.

Compliance with ethics guidelines. The study was conducted according to the principles stated in the Declaration of Helsinki, and approved by the local Ethical Committee of the Area Vasta Nord Ovest of Tuscany region (IRB number 230320). All patients signed a written informed consent.

Data availability. The datasets generated during and/or analyzed during the current study are available from the corresponding author on reasonable request.

Open Access. This article is licensed under a Creative Commons Attribution-NonCommercial 4.0 International License, which permits any non-commercial use, sharing, adaptation, distribution and reproduction in any medium or format, as long as you give appropriate credit to the original author(s) and the source, provide a link to the Creative Commons licence, and indicate if changes were made. The images or other third party material in this article are included in the article's Creative Commons licence, unless indicated otherwise in a credit line to the material. If material is not included in the article's Creative Commons licence and your intended use is not permitted by statutory regulation or exceeds the permitted use, you will need to obtain permission directly from the copyright holder. To view a copy of this licence, visit http://creativecommons.org/licenses/by$\mathrm{nc} / 4.0 /$.

\section{REFERENCES}

1. Coppelli A, Giannarelli R, Aragona M, Penno G, Falcone M, Tiseo G, et al. Hyperglycemia at hospital admission is associated with severity of the prognosis in patients hospitalized for COVID-19: the Pisa COVID-19 study. Diabetes Care. 2020;43: 2345-8.

2. Falcone M, Tiseo G, Barbieri G, Galfo V, Russo A, Virdis A, et al. Role of low-molecular-weight heparin in hospitalized patients with severe acute 
respiratory syndrome Coronavirus 2 pneumonia: a prospective observational study. Open Forum Infect Dis. 2020; 7:ofaa563.

3. Falcone M, Tiseo G, Giordano C, Leonildi A, Menichini M, Vecchione A, et al. Predictors of hospital-acquired bacterial and fungal superinfections in COVID-19: a prospective observational study. J Antimicrob Chemother. 2021;76:1078-84.

4. Mussini C, Falcone M, Nozza SC, Parrella R, Meschiari $\mathrm{M}$, et al. Therapeutic strategies for severe COVID-19: a position paper from the Italian Society of Infectious and Tropical Diseases (SIMIT). Clin Microbiol Infect. 2021;27:389-95.

5. Stebbing J, Sánchez Nievas G, Falcone M, Youhanna $\mathrm{S}$, Richardson $\mathrm{P}$, Ottaviani $\mathrm{S}$, et al. JAK inhibition reduces SARS-CoV-2 liver infectivity and modulates inflammatory responses to reduce morbidity and mortality. Sci Adv. 2021;7:eabe4724.

6. Jones BE, Brown-Augsburger PL, Corbett KS, Westendorf K, Davies J, et al. LY-CoV555, a rapidly isolated potent neutralizing antibody, provides protection in a non-human primate model of SARSCoV-2 infection. BioRxiv. 2020. https://doi.org/10. 1101/2020.09.30.318972.

7. Gottlieb RL, Nirula A, Chen P, Boscia J, Heller B, Morris J, et al. Effect of bamlanivimab as monotherapy or in combination with etesevimab on viral load in patients with mild to moderate COVID-19: a randomized clinical trial. JAMA. 2021;325:632-44.

8. Weinreich DM, Sivapalasingam S, Norton T, Ali S, Gao $\mathrm{H}$, Bhore $\mathrm{R}$, et al. Trial Investigators. REGNCOV2, a neutralizing antibody cocktail, in outpatients with Covid-19. N Engl J Med. 2021;384: 238-51.

9. RECOVERY Collaborative Group, Horby PW, Mafham M, Peto L, et al. Casirivimab and imdevimab in patients admitted to hospital with COVID-19 (RECOVERY): a randomised, controlled, open-label, platform trial. medRxiv 2021.06.15.21258542.

10. ECDC. SARS-CoV-2 variants of concern pose a higher risk for hospitalisation and intensive care admission. https://www.ecdc.europa.eu/en/newsevents/sars-cov-2-variants-concern-pose-higherrisk-hospitalisation-and-intensive-care

11. Hoffmann M, Arora P, Groß R, Seidel A, Hörnich BF, Hahn AS, et al. SARS-CoV-2 variants B.1.351 and P.1 escape from neutralizing antibodies. Cell. 2021;184: 2384-2393.e12.
12. https://www.aifa.gov.it/en/uso-degli-anticorpimonoclonali

13. Wang $P$, Casner RG, Nair MS, et al. Increased resistance of SARS-CoV-2 variant P.1 to antibody neutralization. Cell Host Microbe. 2021;29:747-51.

14. Copin R, Baum A, Wloga E, Pascal KE, Giordano S, Fulton BO, et al. The monoclonal antibody combination REGEN-COV protects against SARS-CoV-2 mutational escape in preclinical and human studies. Cell. 2021;184:3949-61.e11.

15. Planas D, Veyer D, Baidaliuk A, Staropoli I, GuivelBenhassine F, Rajah MM, et al. Reduced sensitivity of SARS-CoV-2 variant Delta to antibody neutralization. Nature. 2021;596:276-80.

16. SARS-CoV-2 increased circulation of variants of concern and vaccine rollout in the EU/EEA, 14th update, 2021. Available at: https://www.ecdc.europa. $\mathrm{eu} /$ sites/default/files/documents/RRA-covid-19-14thupdate-15-feb-2021.pdf.

17. O’Toole Á, Hill V, Pybus OG, Watts A, Bogoch II, Khan $\mathrm{K}$, et al. Tracking the international spread of SARS-CoV-2 lineages B.1.1.7 and B.1.351/501Y-V2. Welcome Open Res. 2021;6:121.

18. ECDC. 2021. https://covid19-country-overviews. ecdc.europa.eu/\#4_Variants_of_concern.

19. Funk T, Pharris A, Spiteri G, Bundle N, Melidou A, Carr $\mathrm{M}$, et al. Characteristics of SARS-CoV-2 variants of concern B. 1.1.7, B.1.351 or P.1: data from seven EU/EEA countries, weeks 38/2020 to 10/2021. Euro Surveill. 2021;26:2100348.

20. SARS-COV-2 Delta variant now dominant in much of the European Region and efforts must be reinforced to prevent transmission, warn WHO/Europe and ECDC. 2021. Available at: https://www.ecdc. europa.eu/en/news-events/sars-cov-2-deltavariantnow-dominant-european-region. Accessed 1 Aug 2021.

21. Istituto Superiore di Sanità. 2021. https://www.iss. it/documents/20126/0/FLASH+SURVEY+Varianti_ SARS-CoV-2_30luglio.pdf/6c1c9969-e62c-cf196d1e-d9679e21692a?t=1627647977533. Accessed 1 Aug 2021.

\section{Publisher's Note}

Springer Nature remains neutral with regard to jurisdictional claims in published maps and institutional affiliations. 